Case Study

\title{
EXTRA-PULMONARY TUBERCULOSIS MIMICKING FRONTAL SINUSITIS
}

\author{
Singh I', Tandon S', Yadav SPS ${ }^{2}$ \\ ${ }^{1}$ Department of ENT, Maulana Azad Medical College, New Delhi, India \\ ${ }^{2}$ Department of ENT, Pgimer, Rohtak
}

\section{ABSTRACT}

Tuberculosis of paranasal sinuses is very rare. It is usually secondary to pulmonary Tuberculosis. Among the Paranasal sinuses, maxillary sinus is the most common to be involved by tuberculosis. Frontal sinus Tuberculosis is very rarely seen. We report a case of extra-pulmonary Tuberculosis of frontal sinus presenting as mucocele in a 40 year old female who presented with a painless swelling. Computed Tomographic scan of Paranasal Sinuses revealed it to be a mucocele but Fine Needle Aspiration Cytology clinched the diagnosis. It was managed conservatively by Anti Tubercular Treatment.

\section{INTRODUCTION}

Tuberculosis (TB) of Paranasal Sinuses (PNS) is rare. The infection usually occurs secondary to pulmonary TB and reaches PNS via hematogenous route or lymphatics. ${ }^{1}$ The early features of TB of PNS are non specific and mimic chronic sinusitis thereby leading to delay in diagnosis and treatment. Diagnosis is challenging and can be confirmed only on histopathological examination. We report an unusual presentation of primary extra-pulmonary TB presenting as frontal sinusitis.

\section{CASE REPORT}

A 40 year old female presented with complaints of painless, progressive swelling over left orbit for 1 year. There were no complaints of headache or recurrent nasal discharge or nasal obstruction. Patient went to private practitioner for the above complaint 6 months back and underwent some surgical procedure, the details of which were not available. On clinical examination, a soft boggy swelling (approx.2x1cm) was palpable in the

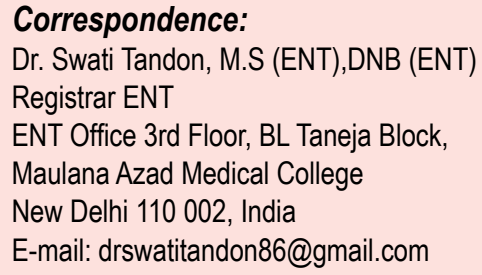

superomedial aspect of left orbit with a healed scar on the overlying skin. The swelling was non tender and aspiration revealed a dry tap. Rest of the ocular examination was unremarkable. Nasal endoscopy was normal. A provisional diagnosis of left frontoethmoidal mucocele was made and CECT PNS was done. CECT PNS and orbit revealed enhancing soft tissue mass in the preseptal region in the superomedial aspect of left orbit with destruction of superomedial wall of left orbit(?from previous surgery). Also, soft tissue opacification of the left frontal sinus was seen (Figures $1 \& 2$ ). Rest of the PNS were normal. FNAC from the swelling was done and was suggestive of features of tuberculosis (Figure 3). CXR was done and did not reveal any pulmonary infiltrates. ESR was raised but Monteux Test was negative. Thus, a diagnosis of primary extra-pulmonary TB of frontal sinus was made and patient was started on Anti-tubercular treatment (ATT) with disappearance of swelling after 6 months of ATT.

\section{DISCUSSION}

With the advent of HIV infection, there has been a rise in extra-pulmonary $T^{1}$.Nasal TB is rare because of structure of the mucosa, respiratory movements of the cilia and bactericidal secretions. However, it can become infected either directly (primarily) through the air current by people sneezing or coughing or by direct inoculation by finger borne infections and by instrumentation. ${ }^{2}$ Primary TB of frontal sinus is rare. 


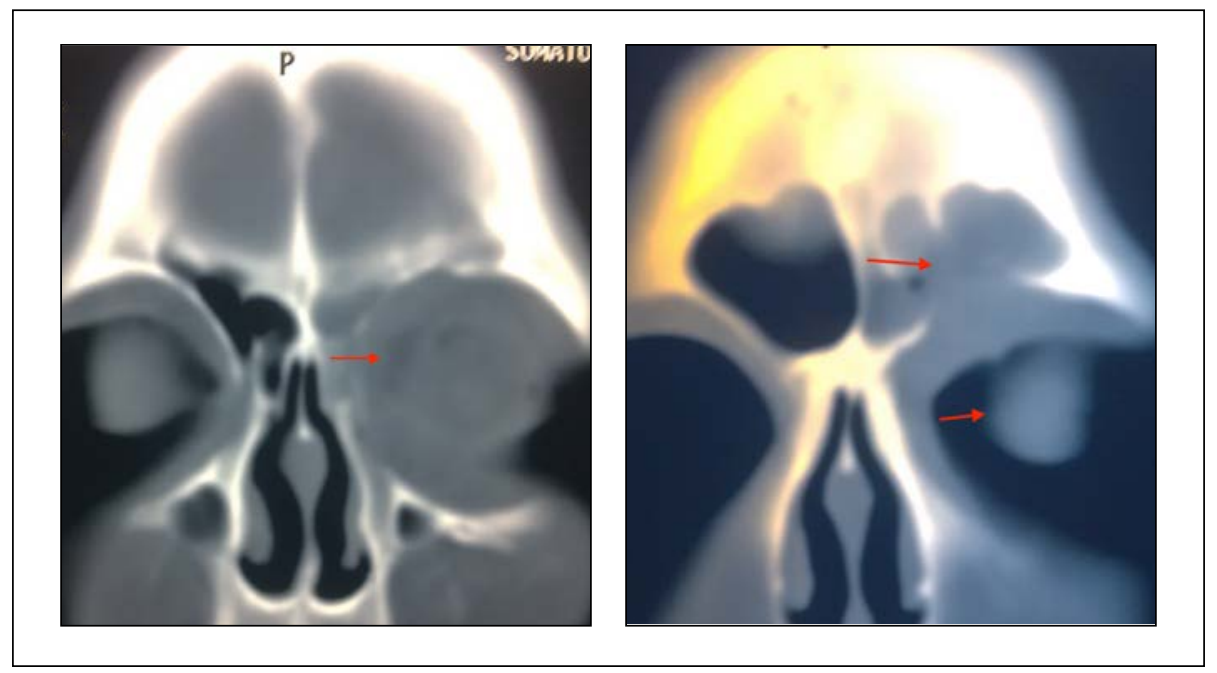

Figure 1 and 2 CT PNS showing soft tissue mass in superomedial aspect of left orbit with opacification of left frontal sinus

Pathologically, three types of sinus TB have been described: In the first type, infection is confined to the mucosa only which presents a boggy, pale appearance. Purulent discharge is minimal, therefore diagnosis is difficult. The second type has bony involvement and fistula formation. Discharge is abundant and the tubercle bacilliare readily found. The third type is hyper-plastic type with the formation of tuberculoma. This is the rarest type. ${ }^{3}$ In our case, the disease was confined to mucosa as seen on CT. The bony defect was due to previous surgery and not a part of disease process.

The most common symptoms of tuberculosis of frontal sinus are: headache, fronto-ethmoid swelling, purulent secretions from nose, deteriorated sense of smell, and nasal bleeding. ${ }^{2}$ Since the early symptoms are non specific, patients are usually treated as chronic sinusitis. ${ }^{4}$ In our case, patient presented with swelling over the left orbit without any nasal complaints. On nasal examination, features suggestive of TB are: crusting, septal ulcer or perforation, granulations in the sinuses ${ }^{5}$ and pale mucosa. In our case, nasal examination was unremarkable. On clinical examination, it was thought to be a mucocele and CT revealed a soft tissue mass.

Diagnosis of TB sinus is challenging. It is based on histopathological examination. Sometimes, immune histochemical stains are required ${ }^{6}$ to establish the diagnosis and differentiate them from other granulomatous conditions. Common differential diagnosis of such lesions includes midline granulomas such as Wegener's granulomatosis, leprosy, sarcoidosis, granulomatous syphilis, rhinoscleroma and rhinosporidiosis.

Treatment of TB PNS is medical with 6 months of ATT. Surgical treatment might be required in late presentations when patient presents with bony fistulae.

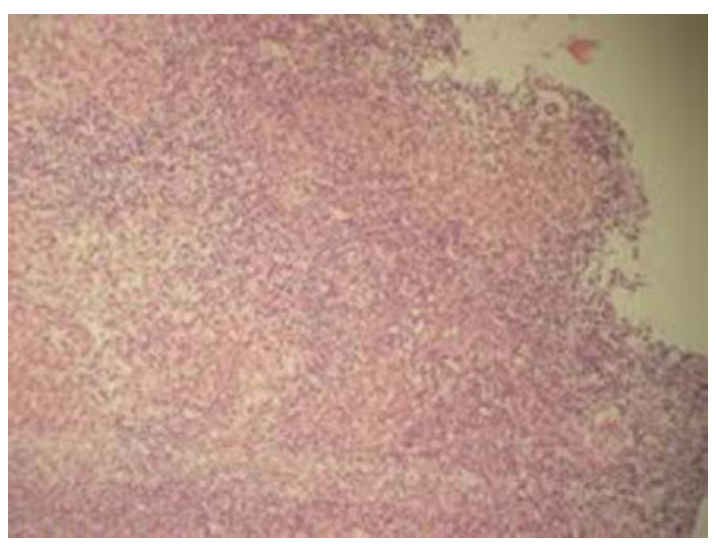

Figure 3 Histopathological Photograph

\section{CONCLUSION}

Frontal sinus TB is very rare. It is essential to have high index of suspicion for diagnosing such cases. Our case highlights the unusual presentation of frontal sinus TB. We also recommend that all samples from nose during surgery or suspicious on endoscopy must be subjected for histopathological examination. 


\section{REFERENCES}

1. Harrison's Principles of Internal Medicine.Vol1.17th Ed.:p1008

2. Dixit $R$, Dave L.Primary Nasal Tuberculosis.Lung India. 2008 ; 25(2): 102-3

3. Sanehi S, Dravid C,Chaudhary N, Venkatachalam VP.Tuberculosis of paranasal sinuses Indian J. Otolaryngol. Head Neck Surg. 2008; 60:85-7

4. Kant S, Srivastava R, Verma AK, Singh HP, Singh S, Ranganath TG et al. Maxillary Sinus Tuberculosis: Various Presentations. Indian J Chest Dis Allied Sci 2013;55:175-7
5. Sethi A, Sareen D, Sabherwal, Agarwal AK. Tubercular Pansinusitis Presenting As a Nasal Polyp.Indian J Tuberc 2005; 52:99-101

6. Budu VA, Bulescu IA, Schnaider A, Popp CG, Toma CL, Mogoantă CA,MühlfayG.A rare case of concomitant tuberculosis of the nose,paranasal sinuses and larynx: clinical, histologicaland immunohistochemical aspects. A case report.Rom J Morphol Embryol 2015; 56:833-836 